\title{
Slow Frequency Hopping for Mitigating Tidal Fading on Rural Long Distance Over-Water Wireless Links
}

\author{
Alex Macmillan \\ School of Informatics \\ The University of Edinburgh \\ Email:s0458553@sms.ed.ac.uk
}

\author{
Mahesh K. Marina \\ School of Informatics \\ The University of Edinburgh \\ Email: mahesh@ed.ac.uk
}

\author{
Jhair Tocancipa Triana \\ School of Informatics \\ The University of Edinburgh \\ Email: j.tocancipa-triana@sms.ed.ac.uk
}

\begin{abstract}
We consider the scenario where long-distance pointto-point wireless links are established over tidal estuaries to bring Internet access to rural communities living in coastal villages. Using real signal strength measurements from links in the Tegola network testbed in northwest Scotland, we highlight the significance of the tidal fading problem, which we reason using a simple geometric model and simulation using the wellknown Pathloss radio link planning tool. We propose slow frequency hopping as a cost-effective, software-only alternative to the commonly used space-diversity approach for mitigating tidal fading, and demonstrate its effectiveness using Pathloss simulation.
\end{abstract}

\section{INTRODUCTION}

For rural and remote areas that are plagued by lack of wired broadband infrastructure, poor mobile phone network coverage, and expensive and high latency satellite coverage, WiFibased long distance wireless mesh networking has emerged in recent years as a cost-effective approach for enabling Internet access to communities (see [1], [2] and references therein). This has led to considerable work from the research community looking at a range of issues, including the understanding of long-distance wireless link characteristics in rural settings, notably by Brewer et al. [3] and Raman et al. [4]. The main conclusion from these link characterization studies is that rural long-distance wireless links experience negligible link quality variations and function as planned with a strong correlation between received signal strength and link quality.

One conclusion from previous work that is of particular relevance to this paper is that long, line-of-sight links in rural networks experience very low multipath interference and delay spread [3]. This conclusion may not always hold. We consider one such exceptional yet common scenario where point-to-point wireless links are deployed at low altitudes over tidal estuaries and as a result, multipath interference from reflections over tidal water can be significant. In regions such as the Scottish Highlands and Islands where most rural communities (representing 90\% of the population, about a quarter million people) live in coastal villages, this can present a major obstacle to reliable Internet service delivery. In fact, there have been media reports of unreliable broadband service

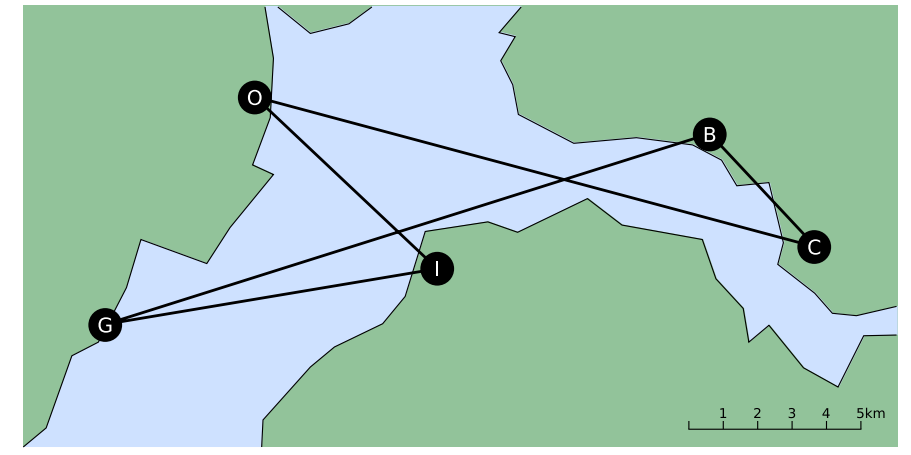

Fig. 1. The six over-water links in the Tegola network.

and ensuing public dissatisfaction with a commercial longdistance wireless deployment in the Western Isles of Scotland that was primarily attributed to wireless signal propagation over tidal waters [5]. The extent of this problem in general can be appreciated by considering areas with tidal estuaries and archipelagos worldwide (e.g., Japan, Indonesia, United Kingdom).

In this paper we revisit wireless transmission over tidal water by showing the significance of the problem using signal strength measurements taken from over-water links in the Tegola network, a long-distance WiFi mesh network testbed deployed in northwest Scotland connecting several remote communities to the Internet [2]. All of the links in the network are at least partly over-water (See Figure 1). We reason about tidal fading effects by developing a simple geometric model. We also perform simulations using a more realistic and wellknown radio link planning tool called Pathloss [6].

More crucially, we propose a slow frequency hopping ${ }^{1}$ technique to mitigate tidal fading. The essential idea is to exploit the frequency dependence of the two-ray interference pattern and adaptively switch to a frequency (channel) that is not currently experiencing fading. Compared to the commonly employed spacial diversity approach, our proposal is

\footnotetext{
${ }^{1}$ Our use of the term frequency hopping is more akin to that of link layer channel hopping techniques such as SSCH [7] and different from that of physical layer frequency hopping in the spread spectrum context.
} 


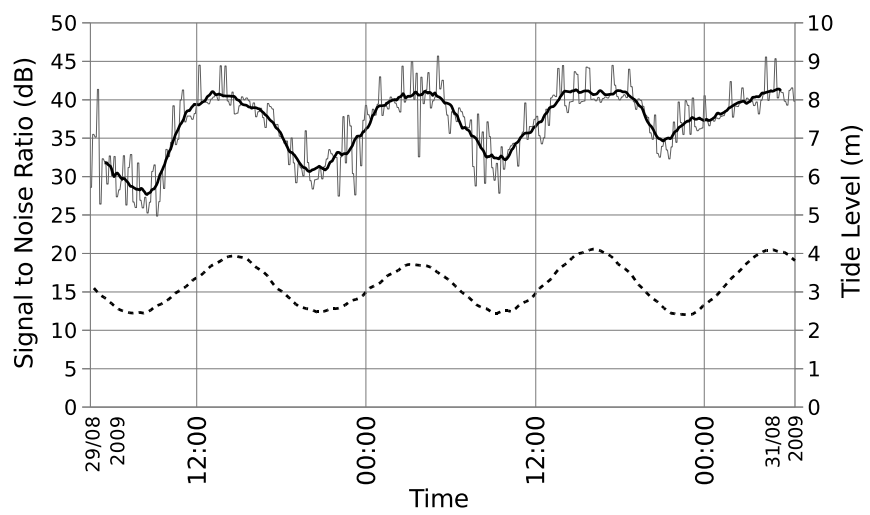

Fig. 2. Signal strength data collected from the Ornsay-Corran link in the Tegola network - the thin (thick) line corresponds to measured signal strength (two hour box-car average). The dashed line shows the tide height data as recorded at a nearby location (Ullapool) for the same time period.

cost-effective as it does not require additional hardware and straightforward to deploy since it is a software-only solution.

The remainder of this paper is organised as follows. In Section II, we explore the tidal fading problem in detail using a geometric model, the pathloss simulation tool and real-world data gathered from the Tegola testbed, we also briefly review related work in the field of over-water wireless communication. Section III describes the commonly used space-diversity solution, and introduces our proposed slow frequency hopping approach. Finally we conclude in Section IV.

\section{THE PROBLEM}

Wireless links over estuaries experience time-varying fading that is dependent on the height of the tide. This is a problem because links experiencing such fading can degrade in quality or fail altogether when the fade margin is insufficient. We have observed this form of fading on almost all point-to-point links in the Tegola network. As an illustration of the tidal fading effect, Figure 2 shows the measured signal strength for the Ornsay-Corran link (between ' $\mathrm{O}$ ' and ' $\mathrm{C}$ ' in Figure 1), a $15.9 \mathrm{~km}$ link almost entirely spanning water between the Isle of Skye and the Scottish mainland. We will use the Ornsay-Corran link in the rest of this paper in reasoning about this problem and the relative merits of various mitigation techniques; this is done for ease of exposition and does not limit the generality of our arguments.

Tidal fading occurs as a result of the interference between the direct line-of-sight path from the transmitter antenna to the receiver antennas and the indirect path that reflects on the water plane (Figure 3). As the tide rises and falls, the length of the reflected path changes. This in turn causes a change in the phase difference between the two paths. As the phase difference approaches 180 degrees, the interference between the paths will be increasingly destructive, resulting in a deeper fade (also referred to as a null). As sea-water acts as a very good reflector at near-grazing angles, the fading due to destructive interference can be significant. In practice, we have recorded fades in excess of $30 \mathrm{~dB}$.

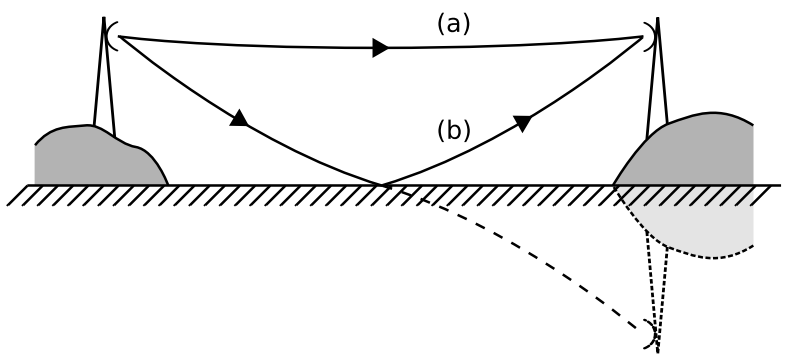

Fig. 3. The two ray model, showing (a) the direct path and (b) the indirect path reflected off the water plane. The reflected path is longer, and so may interfere destructively with the direct path. Refraction is neglected in this diagram. The ray paths are curved due to the flat-earth representation used to clarify the reflection.

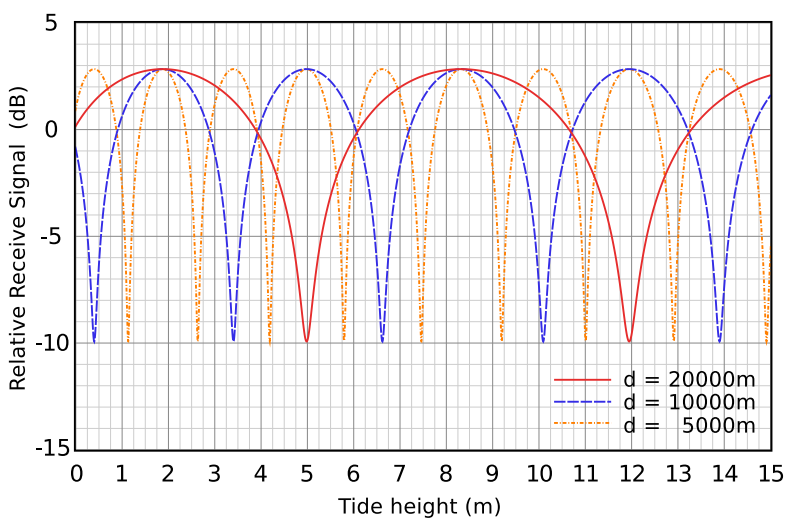

Fig. 4. Relative received signal strength vs. tidal height for links with both antennas $50 \mathrm{~m}$ above sea level calculated using the geometric model with three link lengths. The reflection coefficient is 0.9 . The frequency of operation is $5.180 \mathrm{GHz}$ (at $2.4 \mathrm{GHz}$ the null spacing would approximately double). Atmospheric effects and earth curvature are neglected.

\section{A. Geometric Model}

A simple geometric model can be used to determine the extent of signal fading due to tidal variation. Our model computes the difference in path length between the direct and indirect rays, the resulting phase difference between the rays and thus the amplitude of the received signal relative to the case when only the direct path is present.

Figure 4 shows the relative received signal strength for links of various lengths using our geometric model. The characteristic nulls (deep fades) that occur at specific tide heights are evident from this plot. A link with an insufficient fade margin is likely to fail when it experiences a null. Thus, for such a link, breaks in service can be expected to occur periodically in relation to the rise and fall of the tide.

Further modelling allows us to determine the kind of effects we would expect to observe given the specific tidal height variation. These effects are graphed and explained in Figure 5. In particular, we see that for a given link, the same amount of tidal variation at different tide heights can result in radically different responses in the relative received signal strength (RSS). Note that the measured signal strength for the OrnsayCorran link in the Tegola network shown in Figure 2 can be characterised as a "positive" response. 

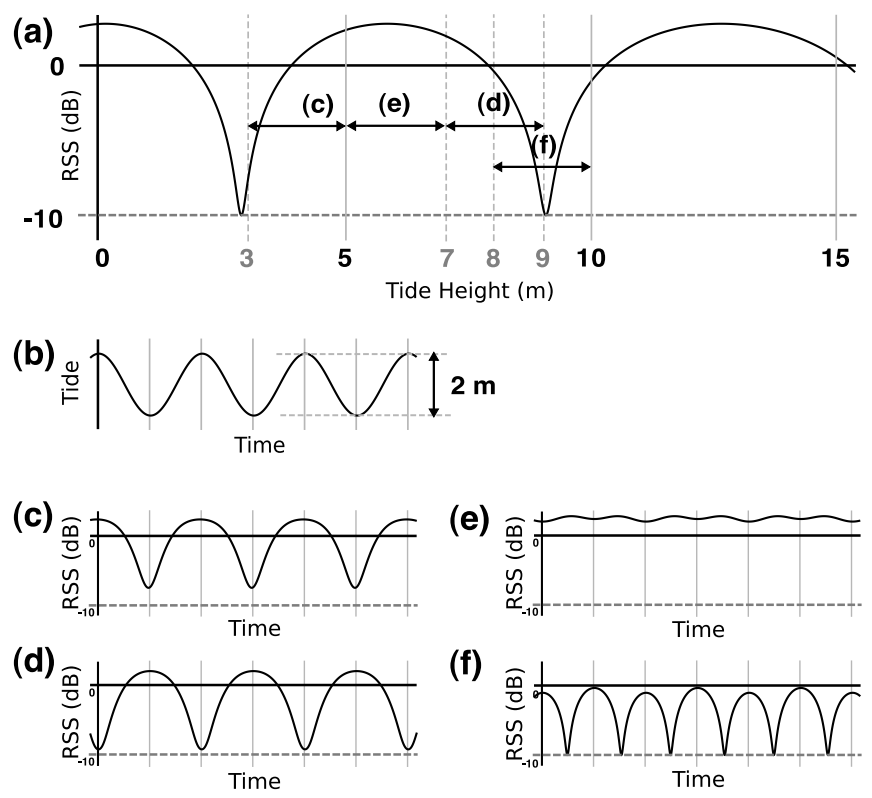

Fig. 5. Modelling the effect of the tide-RSS transfer function on four tidal variations of $2 \mathrm{~m}$. (a) A typical transfer function. (b) The simulated tidal variation. (c) A "positive" response, where increase in tidal height results in increased signal strength. (d) A "negative" response, where increase in tidal height results in decreased signal strength. (e) A "flat" response, where the signal strength is stable to within approximately $2 \mathrm{~dB}$ throughout the tidal cycle. (f) A "cross-null" response, which has two minima for every tidal period.

\section{B. Pathloss Tool}

Now we use a more realistic simulation to demonstrate the effect of tidal variation on the received signal strength. Specifically, we use Pathloss [6], a well-known radio link planning tool from Contract Telecommunication Engineering (CTE). Pathloss accepts input regarding the locations of link endpoints, frequency and mode of operation of the planned link. It uses terrain data to determine the link profile, and then performs calculation using the Longley Rice model [8] in order to determine the expected fading. It also considers environmental factors and earth curvature. We use Pathloss version 4.0.

Figure 6 shows the expected fading with varying tide heights for the Ornsay-Corran link obtained using the Pathloss tool. We see a similar characteristic behavior to that predicted by the simple geometric model. Note that propagation of radio waves is also affected by change in air pressure, which causes rays to diffract. This effect is usually modelled using a $k$-factor, which specifies a gradient change in air pressure with respect to altitude. A k-factor of 1 models no gradient and 1.33 models a "normal" gradient likely in real-world environments. An airpressure gradient has an approximately constant effect on the two-ray model, causing the direct and reflected rays to travel via longer, curving paths. As a result, the shape of the graph shown in Figure 4 will remain essentially the same, though it may be shifted along the horizontal axis. This similarity in shape means that conclusions for the geometric model will still apply under moderate atmospheric conditions. This argument

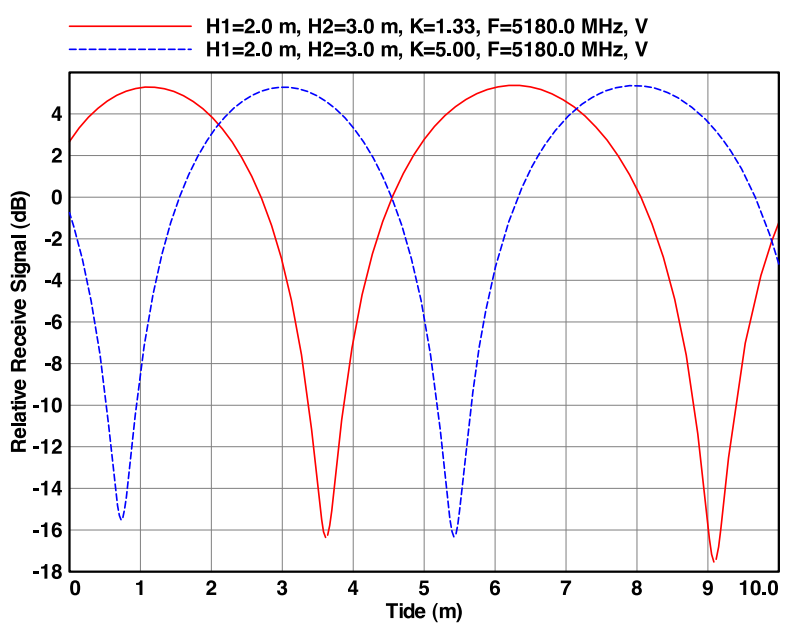

Fig. 6. Signal strength against tide height for the Ornsay-Corran link, simulated using CTE Pathloss 4.0. H1 and $\mathrm{H} 2$ refer to the heights of the antennas above land height, as determined by the link profile - the antenna heights can be very low as they are positioned on the sides of hills.

is validated by the Pathloss simulation of the Ornsay-Corran link for two different k-factor values (see Figure 6).

\section{Related Work}

While many works have studied propagation of microwaves over water, only a small number have studied the effect of variations in water level on signal fading, or the mitigation thereof [9], [10]. Haslett [11] discusses propagation over water, noting the tidal effect on signal strength and suggesting spacial diversity as a common and robust mitigation technique.

Other studies have observed atmospheric conditions such as ducting [12]-[14] or more general disturbances at altitude [15]. Extreme conditions over water can have a radical effect. At low altitudes above the water surface the pressure gradient can invert [12]-[14] causing ducting. When ducting occurs, multiple rays may reach the receiver after several reflections. The phase of the arriving rays is difficult to predict, and the two-ray model becomes invalid. However, ducting is more likely to occur in some climates than in others, and has not been observed to date in the Tegola network.

\section{SOlution APPROACHES}

In this section, we present our proposed frequency diversity based technique for countering tidal fading. We begin by discussing the commonly employed spacial diversity solution to motivate our approach.

\section{A. Spacial Diversity}

Based on our discussion in Section II, we know that multipath interference at the receiver changes with the height of the tide as a result of phase changes between the direct and reflected rays, causing nulls when both rays are out of phase by $180^{\circ}$. In fact, the interference pattern is dependent on the relative height difference between antenna heights and water level (tide height) at any given point in time. Figure 7 shows this relationship for the Ornsay-Corran link for three 


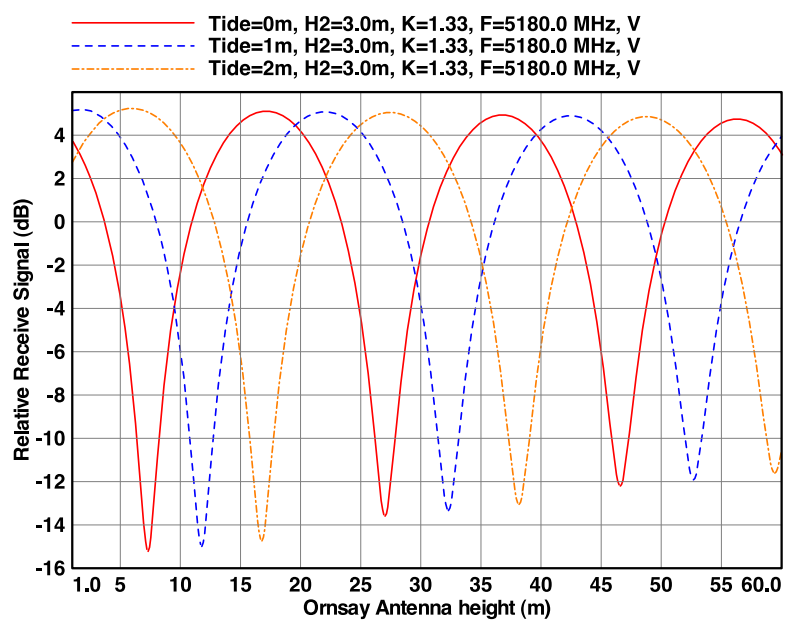

Fig. 7. Path loss simulated using CTE Pathloss 4.0 for varying height of the Ornsay antenna on the Ornsay-Corran link. The graph shows that nulls occur approximately every $20 \mathrm{~m}$.

different tide heights (with respect to the sea level) by varying the antenna height (with respect to the ground level) at Ornsay (assuming it is the receiver). From this figure, we can make a few observations.

Firstly, one could identify an antenna height at the receiver for a given height of the reflecting plane so as to avoid nulls.

Secondly, when the level of the reflecting plane varies, as is the case with tidal water, any specific antenna height will experience a null at some point in the tidal cycle.

Thirdly, the tide height changes merely have the effect of the shifting the signal strength versus antenna height curve along the horizontal axis - separation between nulls and their depth remain practically unchanged.

Finally, the depth of the nulls reduces with the antenna height, which suggests that links with antennas mounted close to the water level are more severely affected by tidal fading.

The commonly used spacial diversity solution to mitigate tidal fading exploits the combined effect of antenna height and tide height on the received signal strength. Specifically, the idea is based on observation (3) above, using two receiving antennas vertically separated by a distance equal to half the separation between the nulls. This ensures that both antennas do not ever experience a null at the same time. As an example, for the Ornsay-Corran link the vertical antenna separation in a spacial diversity system would be around $10 \mathrm{~m}$ as the null separation is approximately $20 \mathrm{~m}$.

While spacial diversity is a robust and popular solution, it has a couple of major drawbacks in terms of deployment complexity and cost, especially from the perspective of community deployed wireless networks in rural areas.

Firstly, employing spacial diversity requires accurate measurement, planning and tools in order to determine the appropriate antenna separation. These may not be readily available to a community looking to install an over-water link.

The second antenna (dish) also substantially increases the overall cost of the installation. For community installations, the

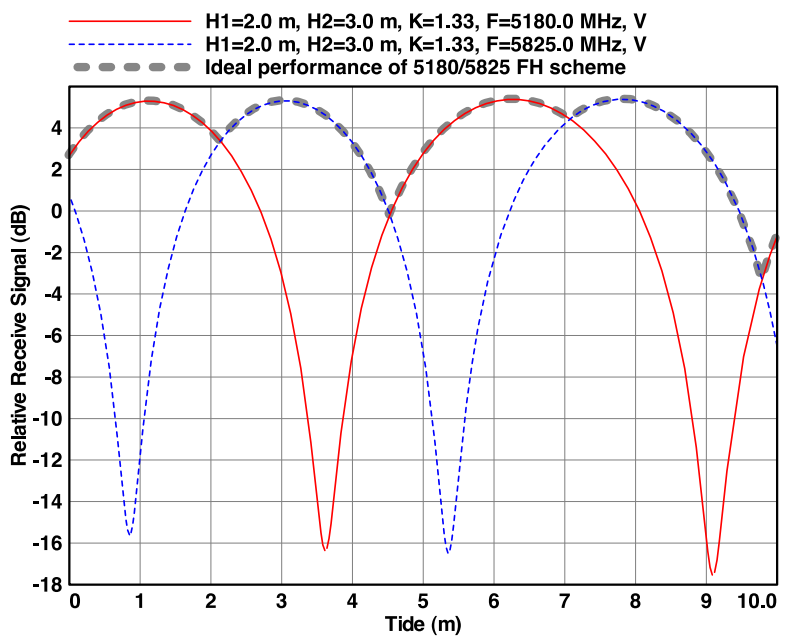

Fig. 8. Modelling of tidal fading when operating at $5180 \mathrm{MHz}$ and $5825 \mathrm{MHz}$ for the Ornsay-Corran link. The fading that would be experienced by a slow frequency hopping system using these two frequencies is also shown.

antenna can be among the most expensive pieces of equipment.

Additionally, the cost of the mast increases rapidly with respect to mast height [16]. A single antenna can be easily and inexpensively mounted just a few metres above the ground, but the cost and complication of installing a mast for an additional $10 \mathrm{~m}$-spaced diversity antenna will be much greater.

\section{B. Our Proposal: Slow Frequency Hopping}

Our proposal, termed Slow Frequency Hopping (SFH), exploits the frequency dependence of the two-ray model by switching channels (frequencies) to avoid nulls. The two-ray model is frequency dependent because the amplitude of the received signal is governed by the phase difference between the direct and indirect rays. As the frequency increases, the wavelength decreases, and so the phase difference will change as well. Thus, while for one frequency the two rays may be $180^{\circ}$ out of phase resulting in a null, at another frequency the two rays will be in phase.

Figure 8 shows potential gains from our SFH proposal, again using the Ornsay-Corran link in the Tegola network as an example. For instance, when operating at $5.825 \mathrm{GHz}$, reflections from a tide height of $3.6 \mathrm{~m}$ would cause a $16 \mathrm{~dB}$ fade. Hopping to $5.180 \mathrm{GHz}$ would instead provide a 4-5 dB enhancement to signal strength due to constructive interference, a net improvement of more than $20 \mathrm{~dB}$. The heavy dashed line shows that an optimal frequency hopping scheme using just these two frequencies would prevent signal degradation due to tidal fading altogether for tides between 0 and $9.4 \mathrm{~m}$ on the Ornsay-Corran link. The ideal frequency separation for this link (where nulls at one frequency are maxima at the other) is approximately $800 \mathrm{MHz}$. Hopping between more than two frequencies is expected to provide further improvement by delivering the 4-5 $\mathrm{dB}$ enhancement over a wider range of tide heights.

Crucially, the SFH technique does not require any additional hardware. The technique can be implemented solely in soft- 
ware by relying upon channel switching features that already exist in virtually all commodity 802.11 wireless radio cards and driver software. Since channel switching in our case needs to only happen at a slower time scale (in the order of minutes at most), channel switching overheads and the ability to switch quickly are non-issues.

Various types of strategies can be employed to realize SFH in practice. A reactive strategy would consider switching to a different channel if the link performance on the current channel becomes unacceptable. An active strategy would continuously measure performance at other channels so as to pick the best channel at a given time (akin to 802.11 rate adaptation algorithms like SampleRate). A proactive strategy would go a step further by incorporating prediction and link modelling to estimate the performance on each channel in the near future.

Note that the available frequencies for switching need to be sufficiently far apart in order to fully realize the benefits of the $\mathrm{SFH}$ technique. This would have been seen to be difficult in the past with the traditional rigid command-and-control spectrum regulatory approach that assigned narrow spectrum bands, each for a specific purpose [17]. With spectrum regulatory reform underway worldwide and technological advances in the form of cognitive radios, a specific spectrum band need no longer be coupled to a specific purpose. As we have demonstrated, even with minor changes to the current regulatory structure, the SFH technique can still be quite effective. Specifically, allowing greater portion of the $5 \mathrm{GHz}$ unlicensed spectrum supported by 802.11a devices for fixed wireless access in rural areas with slightly increased EIRP limits would be sufficient, as 802.11 a devices can hop between 24 non-overlapping $20 \mathrm{MHz}$-wide channels in the $5.15-5.85 \mathrm{GHz}$ range.

In a longer technical report version of this paper [18] we discuss other potential approaches to mitigating the tidal fading phenomenon.

\section{CONCLUSIONS}

\section{A. Preliminary Findings}

Our findings show that there is a significant relation between tide height and signal strength for over-sea wireless links, that deep fades due to tidal effects can have a significant impact on link quality, and that unlike over-land rural networks, tidal effects may be the primary cause of performance degradation.

We propose a software-only solution called slow frequency hopping to effectively mitigate tidal fades without requiring expert knowledge from network installers and maintainers. Our proposed solution can be implemented using features that already exist in all 802.11 devices, and is a cost-effective alternative to the industry solution based on spacial diversity.

\section{B. Planned Work}

We plan to continue to collect data from links in the Tegola network in order to verify conclusions drawn from modelling in this paper. This data will be correlated with available weather information in order to better characterise the effect of adverse conditions such as rain and humidity on the accuracy of the geometric model.

More crucially, we plan to develop a prototype implementation of the proposed slow frequency hopping mitigation technique and conduct a measurement-based evaluation on the Tegola network to experimentally validate our simulation results and compare with the spacial diversity solution. We also plan to design and evaluate reactive, active and proactive frequency hopping strategies.

\section{ACKNOWLEDGMENTS}

This work was supported in part by The Royal Society. We would like to thank Peter Buneman and Mino Bernardi for helpful discussions, and British Oceanographic Data Centre (BODC) for the tidal data.

\section{REFERENCES}

[1] Wireless Networking in the Developing World. http://wndw.net/index.html, Dec 2007. Second Edition.

[2] G Bernardi, P Buneman, and M Marina. Tegola Tiered Mesh Network Testbed in Rural Scotland. In Proc. ACM MobiCom 2008 Workshop on Wireless Networks and Systems for Developing Regions (WiNS-DR'08), Sep 2008.

[3] A Sheth, S Nedevschi, R Patra, S Surana, E Brewer, and L Subramanian. Packet Loss Characterization in WiFi-based Long Distance Networks. In Proc. IEEE Infocom, 2007.

[4] D Gokhale, S Sen, K Chebrolu, and B Raman. On the Feasibility of the Link Abstraction in (Rural) Mesh Networks. In Proc. IEEE Infocom, 2008.

[5] West Highland Free Press. ConCom: Growing Tide of Dissatisfaction, Feb 2008.

[6] Pathloss 4.0, Oct 2009. (Online) http://www.pathloss.com/p4/index.html.

[7] P Bahl, R Chandra, and J Dunagan. SSCH: Slotted Seeded Channel Hopping for Capacity Improvement in IEEE 802.11 Ad-Hoc Wireless Networks. In Proc. ACM MobiCom, 2004.

[8] P Rice and A Longley. Prediction Of Tropospheric Radio Transmission Loss Over Irregular Terrain. A Computer Method, Jan 1968.

[9] A LaGrone, A Straiton, and H Smith. Synthesis Of Radio Signals on Overwater Paths. IRE Transactions on Antennas and Propagation, 3(2):48-52, 1955.

[10] N Fuke, K Sugiyama, and H Shinonaga. Long-range Oversea Wireless Network Using $2.4 \mathrm{GHz}$ Wireless LAN Installation and Performance. In Proc. ICCCN, 2003.

[11] C Haslett. Essentials of Radio Wave Propagation, chapter 4 Reflection, scatter and penetration, pages 95-98. Cambridge University Press, 2008.

[12] J McPetrie, B Starnecki, and H Jarkowski. Oversea Propagation on Wavelengths of 3 and 9 Centimeters. In Proceedings of the IRE, Jan 1949.

[13] T Inoue and T Akiyama. Propagation Characteristics on Line-of-sight Over-sea Paths in Japan. IEEE Trans. Antennas Propag., 22(4):557-565, 1974.

[14] D Siddle, E Warrington, and S Gunashekar. Signal Strength Variations at $2 \mathrm{GHz}$ for Three Sea Paths in the British Channel Islands: Observations and Statistical Analysis. Radio Sci., 42(4):RS4019, Aug 2007.

[15] W Lam and A Webster. Microwave Propagation on Two Line-of-sight Oversea Paths. IEEE Trans. Antennas Propag., 33(5):510-516, 1985.

[16] S Sen and B Raman. Long Distance Wireless Mesh Network Planning: Problem Formulation and Solution. In Proc. World Wide Web (WWW) Conference, 2007.

[17] M Cave, C Doyle, and W Webb. Essentials of Modern Spectrum Management. Cambridge University Press, 2007.

[18] A Macmillan, $M$ Marina, and $J$ Triana. Slow Frequency Hopping for Mitigating Tidal Fading on Rural Long Distance Over-water Wireless Links. The University of Edinburgh, Informatics Research Report EDI-INF-RR-1365, Mar 2010 http://homepages.inf.ed.ac.uk/mmarina/papers/EDIINF-RR-1365.pdf. 